\title{
Análise de requisitos de softwares para implantação em ambientes reais no cenário de Smart Grid
}

\author{
Antônio A. Rodrigues* Artur F. da S. Veloso* \\ Isaac J. C. Lopes* Ricardo A. L. Rabelo* \\ Davi L. de Oliveira ${ }^{* *}$ Alysson R. de F. Santos ${ }^{* * *}$ \\ José E. A. Alencar* \\ * Universidade Federal do Piauí UFPI \\ Teresina, Piauí, Brasil, (e-mail: junioraraujo03@gmail.com, \\ arturfdasveloso@gmail.com, isaack_coutinho@hotmail.com, \\ ricardoalr@ufpi.edu.br, jeduardo824@gmail.com) \\ ** Universidade Federal do Piauí UFPI \\ Picos, Piauí, Brasil, (e-mail: daviluis323@gmail.comr). \\ *** Faculdade Estácio CEUT \\ Teresina, Piauí, Brasil, (e-mail: alyssonramirez@gmail.com).
}

\begin{abstract}
The intelligent electricity network (SG) consists of the union of services that use information and communication technologies (ICTs) to make the current power system more efficient. For this to be possible, an Advanced Measurement Infrastructure (AMI) should be implemented to provide an infrastructure for collecting Smart Meter data (SMs) and send it to the utility. Their standardization and analysis of requirements is little analyzed, and often not even considered by related works. The objective of the present work is to analyze the main requirements of service software for SG in order to provide a model of requirements for researchers and professionals who intend to implement these services in a real scenario. In addition, a software requirements framework for SG with a focus on web and mobile applications for DSM was proposed in the SG scenario.

Resumo: A rede elétrica inteligente (SG), consiste na união de serviços que utilizam tecnologias da informação e comunicação (TICs) para fazer com que o sistema elétrico de potência atual seja mais eficiente. Para que isto seja possível, uma Infraestrutura de Medição Avançada (AMI) deverá ser implementada, para prover uma infraestrutura de coleta dos dados de Medidores Inteligentes (SMs) e enviá-los para a concessionária. A sua padronização e análise de requisitos é pouco analisada, e muitas vezes nem considera por trabalhos relacionados. Pensado nisso, o objetivo do presente trabalho é analisar os principais requisitos de software de serviços para $\mathrm{SG}$, de modo a prover um modelo de requisitos para pesquisadores e profissionais da área, que pretendem implementar estes serviços em um cenário real. Adicionalmente, foi proposta uma estrutura de requisitos de software para SG com foco em aplicações web e mobile para DSM no cenário de SG.
\end{abstract}

Keywords: Software requirements analysis, Infrastructure architecture, Internet of Things, Smart Grid, Smart City.

Palavras-chaves: Análise de requisitos de Software, Arquitetura de Infraestrutura, Internet das coisas, Rede Inteligente, Cidades Inteligentes.

\section{INTRODUÇÃO}

A crescente proliferação de dispositivos inteligentes de diversas escalas, bem como a concentração das tecnologias de informação e comunicação (TICs), vêm convertendo a ideia do contexto de redes tradicionais para Internet da Coisas (Internet of Things - IoT). Diante deste contexto, a IoT ainda envolve uma série de desafios na heterogeneidade de dispositivos, gerenciamento de dados, coleta e tratamento de informações geradas por uma enorme variedade

^ Reconhecimento do suporte financeiro deve vir nesta nota de rodapé. de dispositivos interligados à internet. Devido as preocupações dos consumidores com o constante aumento na conta de energia, a necessidade de melhorar o monitoramento da rede elétrica, praticidade na leitura, fornecimento da conta de energia elétrica e entre outros fatores são cada vez mais presentes na realidade atual.

Com base neste crescente avanço da tecnologia, a Smart Grid (SG) é criada para proporcionar uma infraestrutura de comunicação bidirecional entre a Concessionária de Energia Elétrica (CEE) e as residências, de modo a deixar o sistema de distribuição de energia elétrica mais 
eficiênte. Desta forma, pode-se obter um melhor controle, monitoramento, confiabilidade, segurança e eficiência do sistema elétrico de potência. Neste contexto, encontra-se o Smart Meter (SM), que engloba TICs e outras ferramentas computacionais, permitindo assim o armazenamento de informações sobre a leitura do consumo de energia elétrica residencial. Entretanto, um dos principais desafios da implantação de SMs em SGs, é fazer com que essas tecnologias operem de forma padronizada, seguindo uma infraestrutura avançada e escalável Ghosal and Conti [2019]. A Advanced Metering Infrastructure (AMI), fornece uma infraestrutura padronizada para estes tipos de aplicações, que por sua vez, permite que os sistemas distribuídos possam operar na edge, fog e/ou cloud computing de Sousa et al. [2019], Gilbert et al. [2019].

Contudo, este trabalho consiste no levantamento de requisitos de software, para possibilitar a sua implantação em ambientes reais de uma SG. Levando em consideração fatores importantes que deverão ser considerados, antes que seja implementada. Além do mais, o foco deste trabalho é prover uma abordagem bem detalhada, que possa auxiliar pesquisadores a desenvolver estes tipos de aplicações. Adicionalmente, este trabalho leva em considerações fatores importantes como os requisitos de comunicação, troca de mensagens, banco de dados, servidores, serviços na (Cloud Computing) e entre outros parâmetros que já estarão pré-moldados para futuras implementações. Este artigo está organizado da seguinte forma, na seção 2, estão sendo apresentados os trabalhos relacionados. A descrição da infraestrutura de comunicação da SG é exposta na seção 3. Serão detalhados todos os requisitos a serem analisados, na Seção 4. Na Seção 5, será apresentada a abordagem proposta. Adicionalmente, serão expostos os resultados da implementação na seção $6 . \mathrm{Na}$ Seção 7 , serão feitas as discussões. Finalmente, as conclusões e trabalhos futuros estão apresentados na seção 8 .

\section{TRABALHOS RELACIONADOS}

A literatura, fornece uma variedade de métodos de engenharia de software para auxiliar no desenvolvimento de soluções viáveis para promover a integração na SG. Sendo assim, capazes de capturar e integrar aspectos multidisciplinares da engenharia de software, juntamente com engenharia elétrica e conceitos de computação. Como em Ananthavijayan et al. [2017], os autores propõem uma estrutura de requisitos de software para uma arquitetura de SG com foco em sistemas embarcados. A arquitetura proposta está no modelo multi-camadas, e foi usada para implementar os requisitos propostos, que também são analisados. A análise realizada contempla subsistemas, dispositivos e suas operações. com base na análise feita, os autores concluiram que a arquitetura de software MasterSlave é considerada a melhor, comparada com as várias Unidades de Controle Eletrônico (ECUs) presentes no ambiente de SG. Adicionalmente, os autores afirmam que esta arquitetura é adequada para a conformidade com os padrões de software para SGs, e flexível o suficiente para lidar com elementos de software e desenvolvimentos tecnológicos futuristas.

Uma nova arquitetura para SG baseada no paradigma de Sistemas Definidos por Software (SDSys), foi abordado em
Banuenumah et al. [2017]. A arquitetura proposta simplifica os processos de controle e gerenciamento, abstraindo o controle dos dispositivos de hardware para um sistema centralizado, denominado middleware (camada de controle). O trabalho gira em torno da construção de uma solução abrangente para SGs, integrando todos os controles de armazenamento, segurança e redes IoT com os controles de SG para construir uma arquitetura de SG Baseada em Software Definido. Os autores concluem que, uma arquitetura para SG baseada em SDSys, é mais precisa, confiável, segura e extensível. Já em Patti et al. [2016], uma infraestrutura de software distribuída para serviços de finalidade geral em SG foi apresentada. Os autores apresentam um exemplo de implantação na distribuição de redes, onde mostrou-se que edifícios diferentes podem ser associados a diferentes agregadores que fornecem serviços a vários nós, o que é de fato, a principal força do conceito de middleware. Por fim, o objetivo deste artigo é enfatizar como deverá ser o desenvolvimento de aplicações em tempo real para softwares de SG. Neste trabalho, os autores descrevem arquiteturas e metodologias no desenvolvimento de serviços que funcionam em tempo real, para suportar necessidades específicas deste cenário na SG.

Conforme visto nos trabalhos relacionados, o principal foco é o desenvolvimento de soluções para SG. É importante ressaltar que, os trabalhos apresentados possuem ênfase em problemas reais. Portanto, é mais prudente que seja feita uma análise de requisitos como em Ananthavijayan et al. [2017], antes que eles sejam implementados. Para isso, este trabalho apresenta o funcionamento de uma infraestrutura de SG, e logo em seguida, irá listar todos os principais requisitos a serem avaliados antes do desenvolvimento de softwares para cenários reais na SG.

\section{INFRAESTRTUTURA DA SMART GRID}

A SG implementada de forma padronizada utilizando tecnologias de comunicação em AMI, permite uma comunicação bidirecional entre CEE e residências, conforme apresentado na Figura 1. Conforme apresentada, a aquitetura visa gerar, transmitir e distribuir energia elétrica, de modo que ela chegue com qualidade para as residências. Além disso, possui uma comunicação bidirecional desde a geração até as residências, carros elétricos, eletrodomésticos e entre outros. Dessa forma, os consumidores e a concessionária, poderão ter mais praticidade no monitoramento do consumo, geração e qualidade de energia elétrica, além de fazer todo o controle de pagamentos e despesas por meio de web sites e aplicativos móveis Nair et al. [2017].

As TICs que estarão presentes na SG irão fornecer comunicação bidirecional, segura, escalável, independente de SMs em toda a arquitetura, tornando assim, cada vez mais os consumidores mais interessados pela geração na própria residência de modo devolver de volta para a rede (on-grid) ou utilizar em eletrodomésticos da própria residência (offgrid); engajados e capacitados; e integrados em uma infraestrutura energética modernizada. Portanto, cada casa deve conter um SM para coletar o consumo e enviar para a concessionária. O SM é um dispositivo eletrônico que registra, no mínimo, o consumo de energia elétrica de uma residência e envia essas informações pelo menos diariamente para à CEE Banuenumah et al. [2017]. A AMI 


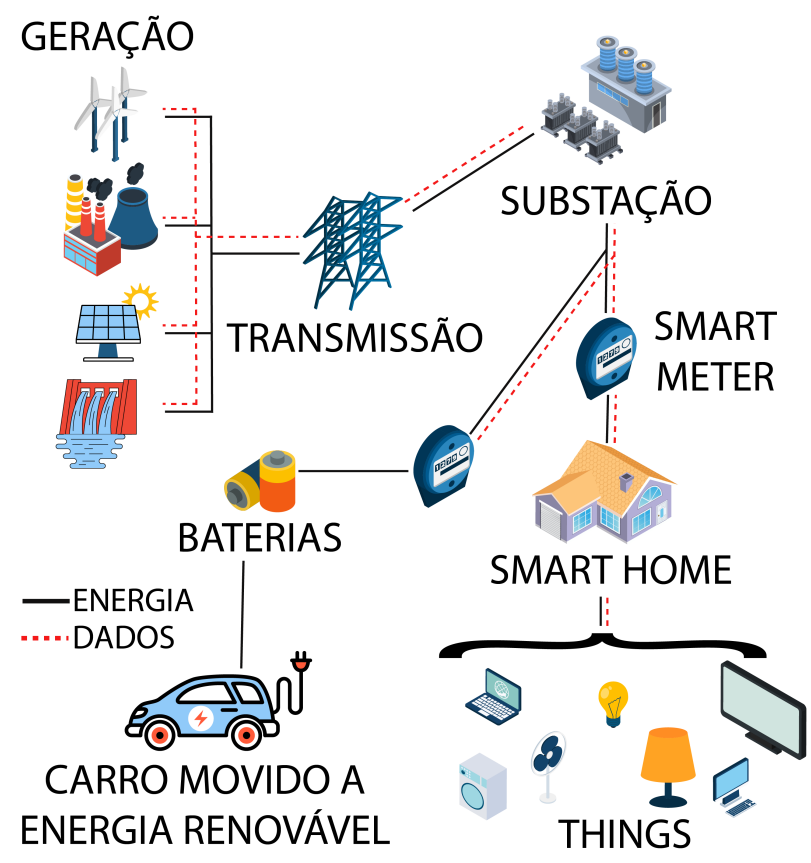

Figura 1. Arquitetura da infraestrutura de comuicação bidirecional de uma SG

difere da tradicional leitura automática de medidores, pois permite que isso seja feito remotamente, sem a necessidade da ida de um funcionário da CEE para fazer manualmente. Esta comunicação pode ser realizada através de conexões fixas com fio ou sem fio. Opções de comunicação com fio incluem portadora de linha de energia (PLC). E sem fio, estão as tecnologias de longa distância como IEEE 802.11, IEEE 802.15.4, LoRa, WiMax, GSM, 3G, 4G, LTE, NBIOT e o $5 \mathrm{G}$.

Aplicações de automação residencial também podem ser aderidos ao cenário de SG, pois a tendência que é cada vez mais, os consumidores invistam nestes tipos de tecnologias. Portanto, já devem ser modeladas considerando todos os principais aspectos da SG, e as reais necessidades prioritárias a sere consideradas em sua implementação Waghmare et al. [2017]. Assim, abrindo portas para a IoT, que a cada ano cresce o número de produtos implementados para estes tipos de aplicação. Portanto, a IoT, suas funcionalidades, segurança, conectividade e demais requisitos, também devem ser modelados e considerados antes de sua implementação. À medida que o uso de energia residencial muda para a eletrificação estratégica e a integração de recursos energéticos distribuídos (DERs) juntamente com tecnologias de IoT, uma casa dinâmica, eficiente e de alto desempenho se torna uma prioridade. Em uma Home Energy Management Systems (HEMS), é otimizado o uso de energia elétrica, com objetivo de economizar, impulsionar a modernização da eficiência energéticas em residências, interagir dinamicamente com a SG e gerenciar novos DERs quando eles estiverem na SG. Os HEMS apresentam aos seus consumidores a oportunidade de gerenciar uma infinidade de sistemas em sua casa, incluindo eletrodomésticos e iluminação, aparelhos inteligentes. Ele atua como um gerenciador das cargas residenciais, permitindo trabalhar de forma integral com algoritmos de automação residencial.

\section{ANÁLISE DE REQUISITOS}

Conforme visto, a infraestrutura de comunicação de uma SG, possui diversas aplicações importantes para que se possa ser aderido em ambientes reais. A engenharia de requisitos concentra-se em especificar mudanças desejadas na etapa de desenvolvimento. Os requisitos de software devem especificar o recurso sem qualquer ambiguidade. Completude de definição é importante, independentemente dos requisitos, descreve a funcionalidade, design, restrições e interfaces. Em geral, um requisito de software deve abordar os seguintes atributos: explicação dos recursos a serem implementados (aplicação real), requisitos de interface, fluxo de dados, frequência de ocorrência, tempo de latência, confidencialidade, atributos de dados, informação de segurança de dados e latência Ananthavijayan et al. [2017]. $\mathrm{Na}$ literatura, existem muitos trabalhos sobre fundamentação de requisitos voltados para Automação Residencial, Internet das Coisas, Smart Cities, SG, SMs e entre outras áreas frequentemente mencionadas na literatura. Entre elas, estão: interoperabilidade, descoberta e gerenciamento de dispositivos, adaptação dinâmica, ciência de contexto, escalabilidade, tratamento de grandes volumes de dados, segurança, gerenciamento de dados e ferramentas de desenvolvimento para aplicações. Pensando nisso, serão analisados os requisitos para o desenvolvimento dessas aplicações. A análise consiste em nível de aplicação de software, infraestrutura de comunicação e dados em uma SG.

\subsection{Interoperabilidade}

A interoperabilidade é uma etapa entre os requisitos para o desenvolvimento de aplicações para SG. A integração de termos como hardware e software, protocolos, dados, são alguns dos principais desafios para a sua concretização. A integração de dispositivos atinge diversos níveis como: baixo nível, no qual é necessário utilizar diversos dispositivos físicos de forma transparente na Edge Computing Samie et al. [2019]. No nível intermediário, é necessário integrar e disponibilizar dados gerados pelos dispositivos para garantir serviços de valor agregados aos usuários. E no alto nível, agregação e transformação dos dados providos pelos dispositivos por uma estrutura padronizada e desenvolvida de forma a permitir que desenvolvedores de aplicações não necessitem ter o conhecimento acerca dos requisitos específicos dos dispositivos físicos da estrutura. Dessa forma, promove a interoperabilidade entre diferentes tecnologias e plataformas.

\subsection{Descoberta e gerenciamento de dispositivos}

A infraestrutura de comunicação deverá ser dinâmica e desconhecida, visto que os dispositivos podem ser integrados aos ambientes utilizados de maneira oportunista e não previamente planejada. A descoberta e o gerenciamento de diversos dispositivos são alguns dos demais requisitos importantes para que uma arquitetura de SG possa funcionar perfeitamente em um cenário real. Assim, permitindo uma integração única de dispositivos que possam ser descobertos e gerenciados, de modo a fornecer informações do seu estado e recebendo comandos de controle. 


\subsection{Adaptação dinâmica}

A adaptação dinâmica é um dos outros requisitos essenciais e importantes para plataformas de serviços de IoT presentes na SG. Pois, diversos dispositivos podem se apresentar e se tornar indisponíveis por diversos motivos, como falha, capacidade energética, indisponibilidade de acesso à rede, ou outras formas de falhas na conexão. Assim, a possibilidade de integração dinâmica, é essencial em aplicações de domínios críticos, onde diversas situações podem apresentar estado crítico de disponibilidade de aplicações em funcionamento. A adaptação dinâmica tem papel importante para manter diversos dispositivos e aplicações funcionando em repleto estado de mudança.

\subsection{Ciência de contexto}

Um dos outros requisitos essenciais deve ser atendido pelas diversas plataformas de serviços para SG, é a ciência de contexto. Este requisito atribui a responsabilidade pela coleta, gerenciamento e processamento de informações de contexto promovido pela crescente fonte de dispositivos. A ciência do contexto, é parte importante para o desenvolvimento de aplicações para SG, de modo a examinar e reagir de acordo com as mudanças de contexto e características de extrema relevância para os ambientes reais de uma SG.

\subsection{Escalabilidade}

A escalabilidade é responsável pelo crescimento pequeno ou grande de usuários utilizando um determinado tipo de serviço. Aplicações para SG necessitam ser escaláveis pois devem integrar uma enorme quantidade de dispositivos interoperáveis. A escalabilidade da Fog Computing, promove o processamento dos dispositivos para mais próximos das aplicações de IoT. Diminuindo a latência e buscando melhorias de taxas de tempo de resposta dos dispositivos para usuários e aplicações. Reduzindo o fluxo de dados enviados diretamente da Edge para a Cloud Computing.

\subsection{Tratamento de grandes volumes de dados}

Devido a grande quantidade de nós contectados a infraestrutura de comunicação, e enviando a todo momento para um servidor central da CEE, esses davos devem ser tratados e organizados de forma padronizada. Pois, a falta de padronização pode ocasionar na perda de dados, ou até mesmo no armazenamento de forma errada. Portanto, o tratamento dos dados é um dos requisistos a ser analisado e bem modelado antes que o sistema esteja em pleno funcionamento em cenários reais. Porque erros neste processo, podem ser irreversíveis.

\subsection{Segurança}

A responsabilidade pela enorme quantidade de dados gerados entre os dispositivos é implementada pela CEE, pois é ela quem se responsabiliza pela infraestrutura de comunicação, logo, deverá se preparar para possíveis ataques ou sequestros de dados. O volume de dados é um requisito que precisa de total atenção para a funcionalidade, pois, possui informações importantes e sigilosas de dados pessoais transitando na AMI Cicirelli et al. [2019]. Vale ressaltar que a segurança tem nível máximo de exigência entre os requisitos de software.

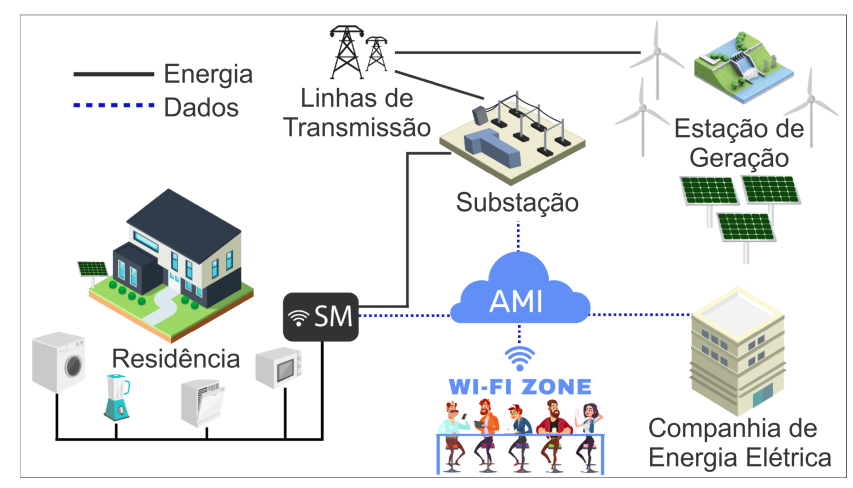

Figura 2. Aquitetura modelada para a abordagem proposta

\subsection{Ferramentas para desenvolvimento de aplicações}

É importante o uso de ferramentas possibilitem a implementação de software de multicamadas, capazes de serem interoperável. Ou seja, que possam ser executados atravéz de web sites ou por qualquer aplicativo móvel. Algumas funcionalidades deverão ser consideradas, como a facilidade de implementações que utilizem ferramentas de IHC (Inteface Humano-computador), possibilitando uma melhor aplicação para o cliente, e uma facilidade no uso das ferramentas, sistemas operacionais não deve ser um impace para a instalação e execução destas aplicações e muito menos o hardware utilizado. No presente trabalho a proposta do trabalho visa a inserção de todos os requisitos estudados e levantado para a validação da proposta da arquitetura de sistemas definidas por software para atuar nos ambientes ou redes inteligentes (Smart Grind). Implica neste trabalho o uso de casa requisitos descrito no trabalho presente em que durante a pesquisa e na elaboração da proposta em que alguns trabalhos se deparamos com vários autores abordando alguns requisitos para prover uma arquitetura de sistemas de software para Smart Grid, já o nosso trabalho reúne todos os tipos de requisitos citado e trabalhos por diversos autores na literatura onde nenhum aborda todos eles os requisitos e nosso trabalho visa a inserção destes requisitos para a construção do nosso modelo de arquitetura de software para ambientes reais da Smart Grind a serem desenvolvidos por base nesta proposta de arquitetura de requisitos necessário que se devem levar em consideração durante o desenvolvimento de software para estes cenários das redes inteligentes (Smart Grind).

\section{ABORDAGEM PROPOSTA}

Para este trabalho, foi estudada uma arquitetura com objetivo de modelar requisitos de software para uma aplicação implementada pelos autores em da S. Veloso et al. [2018]. Na Figura 2, é mostrado a arquitetura da SG completa incluindo a AMI e sua comunicação com os consumidores e CEE. O SM se conecta com a CEE através do AMI, assim como também poderá fornecer informações para serviços na nuvem. É através dele, que o HEM irá coletar vários dados como, o preço da energia naquele intervalo de tempo. Ele irá verificar quais eletrodomésticos que estarão sendo ligado e desligado, e poderá acioná-los em tempo real. Esta abordagem, está ligada ao contexto dos requisitos levantados na sessão anterior. 


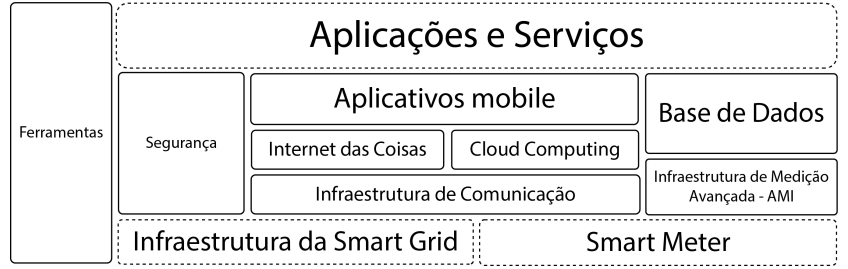

Figura 3. Arquitetura de software proposto

A Figura 3 mostra a arquitetura de software ideal para implantação de aplicações em um ambiente real no cenário de SG. Nela, estão os principais requisitos, e os serviços que serão fornecidos. A arquitetura proposta para este cenário, integra diversos requisitos do trabalho. Cada módulo da arquitetura irá proporcionar e facilitar a comunicação, gerenciamento e segurança do modelo proposto. A troca de informações multiplataforma também deverá ser considerada. Os serviços de gerenciamento de dados na nuvem através da aplicação web ou aplicativos móveis, irá facilitar a exibição de dados para os consumidores e gestores da CEE. Com esta arquitetura, ficará mais fácil estas aplicações numa SG. Pois, os requisitos principais são abordados, e prudentemente obedecidos de maneira a serem utilizados em ambientes reais.

Nesse modelo, são coletadas as informações de consumo da residência pelo SM, e então enviadas para a CEE, onde são monitoradas pelo centro de controle. E logo em seguida, os dados são armazenados na nuvem via internet. Através da API, esses dados são migrados, e transformados em informações que serão fornecidas e apresentadas aos usuários do sistema. A arquitetura de automação residencial nesta abordagem faz a utilização dos seguintes requisitos levantados: interoperabilidade, descoberta e gerenciamento de dispositivos, escalabilidade, tratamento dos dados e gerenciamento e adaptação dinâmica do contexto proposto, onde está dividida em 4 partes, 1) Things (Coisas), é a parte que representa o Hardware da aplicação. Esses things, poderão trocar informações através de dois protocolos mais utilizados, ou via internet (IEEE 802.11) ou via rede local Radio Frequência (IEEE 802.15.4), que por sua vez, é o protocolo mais utilizado na área de IoT. 2) O HEMS irá mostrar, de forma visual, como por exemplo, informações da qualidade dos eletrodomésticos, anomalias na redes, consumo individual, e entre outras informações que poderá auxiliar bastante o consumidor. Na sua estrutura, utiliza o protocolo MQTT para efetuar a integração entre todos os componentes (IoT, serviços na nuvem). E poderá utilizar também o IEEE 802.15.4 para comunicação com os dispositivos de IoT. 3) Serviços na Web, presentes na Cloud Computing, é onde está a base de dados e os serviços implementados. Ele se comunica com o HEMS através do procolo Broker MQTT. E troca informações com aplicativos mobile via HTTP, SOAP ou até mesmo o Broker MQTT. 4) aplicativos mobile, que troca informações com os serviços na nuvem, para prover e apresentar na tela do celular, numa melhor forma para o usuário. Para este trabalho o SM foi implementado com sensores de tensão e corrente para a mensuração do consumo, possui comunicação bidirecional com a CEE, através do protocolo IEEE 802.15.4 e possui encriptação dos dados para evitar roubos de dados via infraestrutura de comunicação.
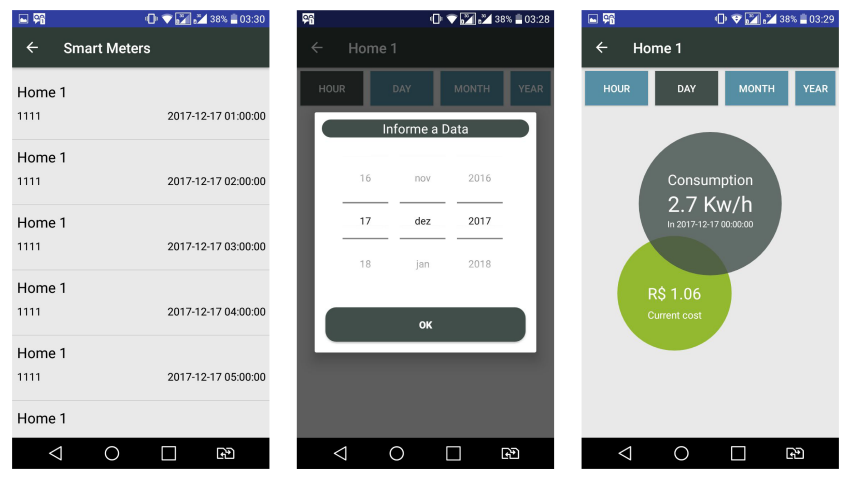

Figura 4. Aplicativo móvel fornecido para cada consumidor

Conforme apresentado na Figura 4, o app mobile foi implementado para apresentar de forma gráfica o consumo, e o valor a ser pago pelo consumidor. A interface do aplicativo é modelado conforme os requisitos expostos anteriormente. O objetivo desta aplicação é auxiliar o consumidor no entendimento da informação do consumo de energia de sua residência através da internet. Desta forma, o consumidor tem acesso de qualquer lugar do mudo, e pode facilmente alterar o seu perfil de consumo para poder economizar em sua fatura. Por fim, a proposta também prover uma interface implementada onde, o HEM informará aos consumidores sobre as informações de consumo de energia de cada aparelho, medição da energia consumida, além de informações que são obtidas facilmente pelo software implementado com base nos requisitos analisados. Que por sua vez, está ligado diretamente ao contexto dos requisitos de "ferramentas desenvolvidas de aplicações para este cenário"e na "descoberta e gerenciamento dos dispositivos", são os principais requisitos encontrados nesta aplicação.

\section{TESTES E DISCUSSÕES}

Os testes da arquitetura proposta, foram rigorosamente seguidos pelos requisitos do modelo de implementação do web site, aplicativo mobile e SM. O teste foi realizado durante um dia, de $00 \mathrm{~h}$ até às $23 \mathrm{~h} 59$, com intervalos de leitura a cada $1 \mathrm{~h}$, correspondendo a 24 amostras coletadas duantes $24 \mathrm{~h}$ de teste. Foram implementados três SMs, um para cada casa. Foi realizada a medição e leitura, envio dos dados para o web site do AMI, que recebeu estes dados e plotou na aplicação mobile, com objetivo de apresentar para os consumidores. Com base nos resultados obtido, foi possível notar que a aplicação funcionou conforme o esperado. Apresentou uma comunicação íntegra, impossibilidade de perda de dados e segurança da informação. Além de apresentar, uma plotagem de dados, para os usuários visualizarem, de uma maneira mais fácil do que nos contadores atuais. Por fim, na tabela 1, foram comparados os trabalhos do estado da arte com o presente modelo proposto considerando os requisitos que são atendidos por cada. Onde, pode-se observar que o requisito é completamente atendido no testbed implementado por este trabalho. Sendo que o símbolo -"representado que o $\mathrm{X}$ presenta o o requisito atendido, e -"representa que o requisito não foi atendido, ou foi parcialmente atendido.

De acordo com a tabela 1, onde o modelo proposto foi comparado com trabalhos da literatura considerando os principais requisitos para implementação de softwares em 
Tabela 1. Comparativo de Requisitos entre Plataformas de Software para Smart Grid

$\begin{array}{lrrrrr}\text { Requisitos } & {[5]} & {[15]} & {[2]} & {[14]} & \text { Proposta } \\ \text { interop. } & - & \mathrm{X} & \mathrm{X} & \mathrm{X} & \mathrm{X} \\ \text { descoberta dispo. } & - & \mathrm{X} & \mathrm{X} & - & \mathrm{X} \\ \text { adaptação din. } & - & - & \mathrm{X} & & \mathrm{X} \\ \text { ciência de cont. } & - & & - & - & \mathrm{X} \\ \text { escalabilidade } & - & \mathrm{X} & - & - & \mathrm{X} \\ \text { trata. de dados } & \mathrm{X} & - & - & & \mathrm{X} \\ \text { segurança } & \mathrm{X} & \mathrm{X} & \mathrm{X} & - & \mathrm{X} \\ \text { geren. de dados } & - & \mathrm{X} & - & - & \mathrm{X} \\ \text { Ferram. aplicações } & \mathrm{X} & \mathrm{X} & \mathrm{X} & \mathrm{X} & \mathrm{X}\end{array}$

cenários de SG. Os trabalhos relacionados, serviram como base para o levantamento de requisitos. Entretanto, os demais trabalhos relacionados não consideram todos o os principais requisistos para propor uma solução padronizada e que possa ser implementada em cenários reais. O modelo proposto se saiu bem, em todos os requisitos defindidos como base de implementação do software para este cenário, diante da investigação dos trabalhos, pode-se encontrar diversas situações reais, como a falta de interoperabilidade, descoberta e gerenciamento de dispositivos, adaptação dinâmica, ciência do contexto, escalabilidade, segurança e gerenciamento de dados. Foram coletados os principais requisitos considerados nos trabalhos, para formação de base do modelo proposto, assim pode-se observar vários desafios que buscamos desenvolver e implementar a validação da proposta deste artigo. A arquitetura proposta utiliza todas as características dos trabalhos relacionados conforme apresentado na tabela 1. Os problemas encontrados nos trabalhos presentes na literatura foram analisados e apresentados mecanismos que preenchem os requisitos para viabilizar a implementação destas soluções em ambientes reais.

\section{CONCLUSÕES E TRABALHOS FUTUROS}

O presente trabalho consiste no levantamento de requisitos de software, para possibilitar a implementação de aplicações para SG. O objetivo é apresentar os principais requisitos que estes softwares precisarão atender para que possam ser implantados em ambientes reais. Este trabalho apresenta os principais requisitos, e modelos de implementação que poderão ser feitos para implantação e testes em ambientes reais. Os estudos foram feitos, e um testbed foi implementado de modo a ser comparado com trabalhos presentes no atual estado da arte. Assim, o modelo proposto conseguiu obedecer aos requisitos, e atendeu ao que o cenário de SG necessita, obtendo um modelo bem mais padronizado comparado com os trabalho discutidos. Podendo assim, ser um dos modelos de padronização para o desenvolvimento de software neste contexto. Contudo, foram implementados o web site da CEE, o protótipo do SM e o aplicativo móvel. Todas as aplicações implementadas obedeceram aos principais requisitos, e apresentaram um funcionamento conforme o esperado. Os dados de consumo foram lidos pelo SM, enviados a nuvem e armazenados no banco de dados da CEE. Os serviços disponibilizam os dados personalizados para o aplicativo do consumidor, através de meios de segurança como autenticações nas requisições. Os dados foram plotados de forma simples e de fácil compreensão, assim tornando mais fácil de ser usado e aderido em ambientes reais.

Como trabalhos futuros, pretende-se integrar estes aspectos de requisitos para o desenvolvimento de aplicações que possam se integrar com o web site da CEE e o aplicativo móvel do consumidor que foram implementados neste trabalho. Adicionalmente, pretende-se incluir a medição de água e gás na arquitetura modelada.

\section{REFERÊNCIAS}

[1] Ananthavijayan, R., Karthikeyan, S.P., Raglend, I.J., Edward, J.B., and Kumar, K.S. (2017). Requirement framework of smart grid software architecture. In High Voltage Engineering and Power Systems (ICHVEPS), 2017 International Conference on, 418-423. IEEE.

[2] Banuenumah, W., Sekyere, F., and Dotche, K. (2017). Field survey of smart metering implementation using a simple random method: A case study of new juaben municipality in ghana. In 2017 IEEE PES PowerAfrica, 352-357. IEEE.

[3] Cicirelli, F., Guerrieri, A., Mastroianni, C., Spezzano, G., and Vinci, A. (2019). The Internet of Things for Smart Urban Ecosystems. Springer.

[4] da S. Veloso, A.F., Rodrigues, A.A., Sobral, J.V.V., Rodrigues, J.J.P.C., Feitosa, M.S.S., and Rabelo, R.A.L. (2018). An IoT Smart Metering Solution Based on IEEE 802.15.4. 2018 IEEE Global Communications Conference (GLOBECOM), 1-6. doi: 10.1109/GLOCOM.2018.8647795.

[5] de Sousa, J.V., Coury, D.V., and Fernandes, R.A. (2019). A survey on cloud computing applications in smart distribution systems. Electric Power Components and Systems, 1-16.

[6] Ghosal, A. and Conti, M. (2019). Key management systems for smart grid advanced metering infrastructure: A survey. IEEE Communications Surveys 8 Tutorials.

[7] Gilbert, G.M., Naiman, S., Kimaro, H., and Bagile, B. (2019). A critical review of edge and fog computing for smart grid applications. In International Conference on Social Implications of Computers in Developing Countries, 763-775. Springer.

[8] Nair, A.S., Ranganathan, P., Salehfar, H., and Kaabouch, N. (2017). Uncertainty quantification of wind penetration and integration into smart grid: A survey. In 2017 North American Power Symposium (NAPS), 1-6. IEEE.

[9] Patti, E., Syrri, A.L.A., Jahn, M., Mancarella, P., Acquaviva, A., and Macii, E. (2016). Distributed software infrastructure for general purpose services in smart grid. IEEE Transactions on Smart Grid, 7(2), 1156-1163.

[10] Samie, F., Bauer, L., and Henkel, J. (2019). Edge computing for smart grid: An overview on architectures and solutions. In IoT for Smart Grids, 21-42. Springer.

[11] Waghmare, P., Chaure, P., Chandgude, M., and Chaudhari, A. (2017). Survey on: Home automation systems. In 2017 International Conference on Trends in Electronics and Informatics (ICEI), 7-10. IEEE. 